\title{
Applications of synchrophasor technologies in power systems
}

\author{
Muhammad Usama USMAN $^{1} \mathbb{1}$, M. Omar FARUQUE ${ }^{1}$
}

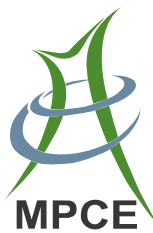

\begin{abstract}
Synchrophasors are time-synchronized electrical measurements that represent both the magnitude and phase angle of the electrical sinusoids. Synchrophasors are measured by fast time-stamped devices called phasor measurement units (PMUs) to constitute the basis of realtime monitoring and control actions in the electric grid. Due to its enhanced situational awareness capabilities, many applications of PMUs are presented in the literature in the past decades. This paper presents a comprehensive summary of synchrophasor technology, its architecture, optimal placement techniques and its applications in electric power transmission and distribution systems. These applications include wide-area situational awareness and monitoring, state estimation, fault location and protective relaying, islanding detection etc. This review also covers some of the existing challenges in its implementation and its potential applications.
\end{abstract}

Keywords Phasor measurement unit (PMU), Supervisory control and data acquisition (SCADA), Synchrophasor technology (ST), Power system

CrossCheck date: 18 July 2018

Received: 11 December 2017/Accepted: 18 July 2018/Published online: 26 October 2018

(C) The Author(s) 2018

$\bowtie$ Muhammad Usama USMAN

musman@fsu.edu

M. Omar FARUQUE

faruque@caps.fsu.edu

1 Department of Electrical and Computer Engineering, Center for Advanced Power Systems, Florida State University, Tallahassee, USA

\section{Introduction}

The modern power system (PS) is a complex interconnected network which can generally be split into four important parts: generation, transmission, sub-transmission, distribution and loads. Due to the complex nature of PS, it is necessary to continuously monitor and protect its elements in order to avoid major contingencies. As a result of different PS contingencies that have occurred in the past, the concept of monitoring the PS network in real-time (RT) has become very important today [1]. Traditionally, supervisory control and data acquisition (SCADA) based monitoring is used for PS but it is unable to capture RT dynamics of the system due to its low resolution, unsynchronized measurements of system parameters and incomplete information of the system behavior. Various cascaded outages occurred in the U.S. in the last two decades were a result of deviation in power flow, frequency, and voltage [2]. Incomplete monitoring resulted in misoperation of protection devices and improper control techniques which leads to millions of customer out of electricity and billions of dollars of losses in the economy [3]. These outages made it necessary to monitor PS parameters such as frequency, phase angle, and amplitude to ensure those system parameters are always within the limits. Hence, in the after effect in 2007, the U.S. Department of Energy in collaboration with Electric Power Research Institute, along with several electric utilities and research institutes formed the North American Synchrophasor Initiative (NASPI) with the role to enhance PS visibility and reliability through wide-area measurement and control, by promoting the use and capabilities of synchrophasor technology (ST) [4, 5].

ST has many applications in electric PS which can be primarily grouped into three categories $[6,7]$ : real-time 
grid operations; system planning and analysis; responsebased control applications.

This paper focuses on an in-depth review of RT grid applications of ST. These applications support RT grid operations by providing wide-area visualization and situational awareness. The paper is organized as follows. In Section 2, the definition of phasor measurement unit (PMU), ST and their importance in PS is provided. In Section 3, the architecture of ST, IEEE standards, and different placement algorithms are discussed. Section 4 covers a detailed summary of many PMU applications in power transmission and distribution systems along with potential research areas. Section 5 concludes the discussion with a comprehensive list of literature to provide further details to the readers.

\section{Synchrophasor technology}

\subsection{Basic principle}

As stated by Charles Steinmetz in 1893, a phasor is a complex counterpart of a sinusoidal wave quantity such that the complex modulus is the cosine wave amplitude, and the complex angle is the cosine wave phase angle [8]. According to him, a sinusoidal waveform that can be represented by:

$x(t)=X_{m} \cos (\omega(t)+\phi)$

can also be represented as the phasor as shown in (2):

$X=\frac{X_{m}}{\sqrt{2}} e^{\mathrm{j} \phi}=\frac{X_{m}}{\sqrt{2}}(\cos \phi+\mathrm{j} \sin \phi)=X_{r}+\mathrm{j} X_{i}$

where $X_{m} / \sqrt{2}$ is the RMS value of the magnitude of the waveform; subscripts $r$ and $i$ are the real and imaginary parts of the complex value and $\phi$, the phase angle depends on the timescale, particularly where $t=0$.

Taking this concept, IEEE standard C37.118.1-2011, defines synchrophasor representation of the signal $x(t)$ in (1) is the value $X$ in (2) where $\phi$ is the instantaneous phase angle relative to a cosine function at the nominal system frequency synchronized to coordinated universal time (UTC). Synchrophasor is defined as "the magnitude and angle of a cosine signal of voltages and currents as referenced to an absolute point in time" as shown in Fig. 1 [9].

\subsection{PMU}

A PMU is an electronic device that provides synchrophasors and frequency measurements for a three-phase $\mathrm{AC}$ voltage and/or current waveforms. Figure 2 shows a functional block diagram of PMU [10]. PMU uses state-of-

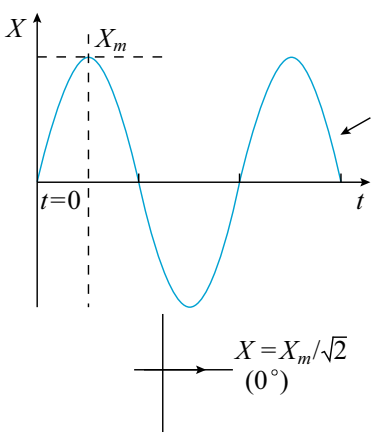

(a)

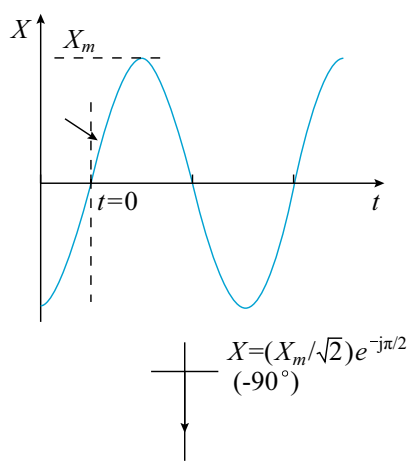

(b)
Fig. 1 Convention for synchrophasor representation

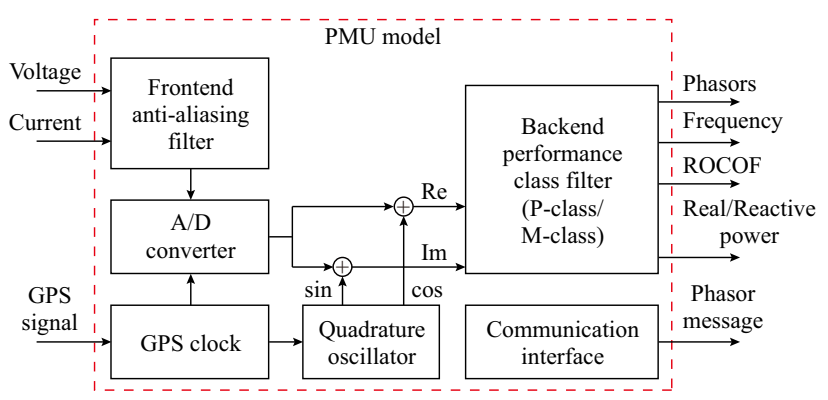

Fig. 2 Functional block diagram of PMU model

the-art digital signal processors that can measure single phase and three phase AC waveforms of time synchronized electrical quantities i.e, voltages $(V)$ and current $(I)$. Inside a PMU, the $V$ and $I$ signals are passed through a front-end anti-aliasing filter to eliminate high-frequency interference signals and then digitized using an A/D converter. For digitization, a fixed sampling rate is used with a synchronized GPS clock. The digitized signals are then processed through complex multiplication with the nominal frequency carrier in order to get the real and imaginary components of the phasor. These components are then passed through a back-end performance class filter (P-class or M-class finite impulse response filter) which gives the required accuracy under dynamic conditions [10].

Final values are used to estimate secondary quantities such as voltage and current phasors (synchrophasors), phase angle difference $\phi$, frequency, the rate of change of frequency (ROCOF), real and reactive power etc.

\subsection{Why ST?}

Before ST, SCADA was used to monitor the PS but SCADA based measurements are traditionally based on steady-state power flow analysis and therefore, cannot observe the dynamic characteristics of the PS. Some key differences between SCADA and PMU measurements are presented in Table 1. 
Table 1 Comparison between PMU and SCADA

\begin{tabular}{lll}
\hline Attribute & SCADA & PMU \\
\hline Resolution & 1 sample every $2-4 \mathrm{~s}$ & $10-60$ samples/s \\
Observability & Steady state & Dynamic \\
Measurements & $\mid V l, I$ & $|V|, \delta, I$, frequency, ROCOF \\
Synchronization & No & Yes \\
Phase angle & No & Yes \\
Focus & Local monitoring and control & Wide area monitoring and control
\end{tabular}

Due to the added advantage of phase angle difference, time synchronization, and fast data rate, PMUs deployment in U.S grid is increasing rapidly. In 2009, there were only 200 research-grade PMUs networked across North America. Currently, there are almost 2500 production-grade PMUs deployed across the U.S. and Canada [11]. These PMUs are able to transmit data to different regional data concentrators for RT wide-area situational awareness.

\section{Architecture, standards and placement of ST}

\subsection{ST architecture}

ST usually implies the use of synchrophasor data from PMUs for PS monitoring. It involves many sensitive instruments to communicate PMU data with the rest of the grid and then to send the data for different applications. ST can be categorized into three layers as shown in Fig. 3 [12].

\subsubsection{Measurement layer}

Measurement layer consists of current transformers (CTs) and potential transformers (PTs), analog units and PMUs. PMUs have a GPS with them to time stamp the data and they are employed in substations to collect the analog data from CTs and PTs.

\subsubsection{Data collection layer}

After collecting the data, PMUs send the data to phasor data concentrators (PDCs). PDC is a device that combines data from several measurement devices. They receive phasor measurements from remote PMUs through a communication medium and stores data in a database management system. The function of the PDC is data handling, synchronization, processing, and storage. PDC through its monitoring system also provides information on the system's performance parameters like latency, data quality, frame rate etc [13].

In a normal configuration, PMUs connect major substations in transmission systems and transmit the measurements in RT over the internet or other communication media such as fiber-optic. These measurements are collected by a local PDC where the data is aggregated in a database management system. Local PDCs belonging to different utilities can also be connected to a centralized PDC.

\subsubsection{Wide area measurement system (WAMS) application / energy management layer}

This is the part of the ST through which PDC sends data to control rooms through communication channels. Since data is provided in RT (neglecting the transmission latency), it provides the RT scenario of the grid. Applications are developed to use that data in order to provide a better visibility of the system.

\subsection{IEEE standards for ST}

The first standard for ST was published as IEEE standard 1344-1995. It specifies synchronization to UTC, time accuracy and waveform sampling requirements. This standard did not include measurement requirements or data hierarchy. It was superseded by IEEE standard C37.1182005 which contains both features. However, a major breakthrough was developed in 2009 when IEEE started a joint project with IEC to harmonize RT communications defined in C37.118 with the IEC 61850 communication standard C37.118-2005 evolved into C37.118-2011 with sub-standards C37.118.1-2011 (measurement of synchrophasors) and C37.118.2-2011 (communication of synchrophasors). Amendment to IEEE Standard C37.118.1-2011 was made to include the guide for PDC requirements for power system protection (PSP), control, and monitoring. Details about these standards can be found 


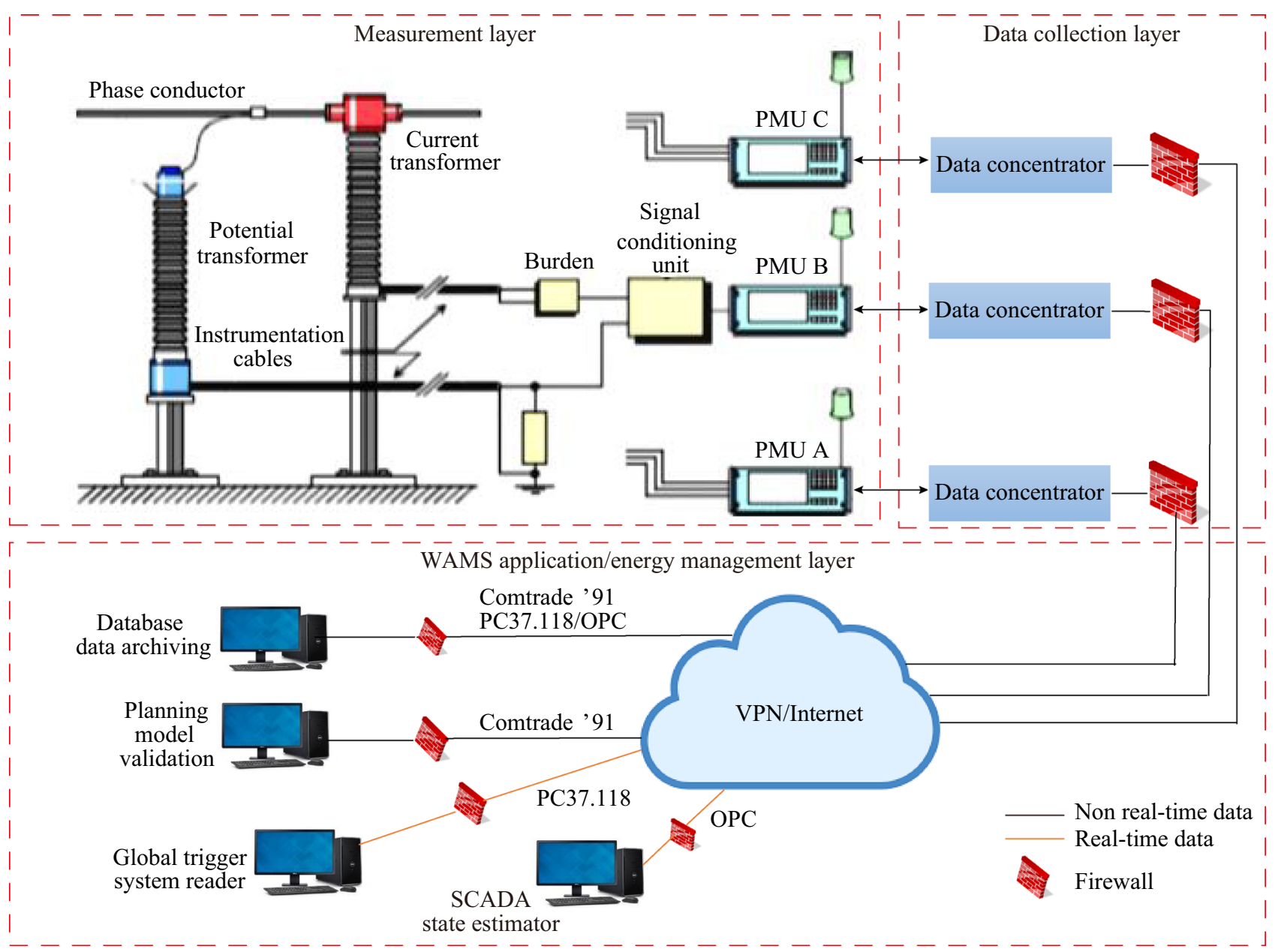

Fig. 3 ST infrastructure

Table 2 IEEE synchrophasor standards

\begin{tabular}{|c|c|c|}
\hline IEEE standards & Description & Features \\
\hline C37.118-2005 & Replaced IEEE 1344 & $\begin{array}{l}\text { Included performance requirements for measurement, communication } \\
\text { adaptable to networks. Basis for current methods and standards }\end{array}$ \\
\hline C37.118.1-2011 & Synchrophasor measurement & $\begin{array}{l}\text { Define frequency and ROCOF measurement under all operating conditions, time } \\
\text { tag and synchronization requirements }\end{array}$ \\
\hline C37.118.2-2011 & Synchrophasor data transfer & Define message types, data types, contents, format and use \\
\hline C37.242-2013 & Guide for synchronization & Guidance for synchronization, calibration, testing, and installation of PMUs \\
\hline C37.244-2013 & PDC guide & Guide to terminology and operation of phasor data concentrators \\
\hline C37.247-2013 & PDC standard & Standardize requirements from concepts in C37.244 \\
\hline
\end{tabular}

in $[8,9,13]$ and [14]. A comprehensive summary of synchrophasor standards is formulated in Table 2.

\subsection{PMU placement}

Work on PMU placement problem started as early as 1993, just after the first idea of this technology came [15].
Optimal PMU placement (OPP) means that how many minimum numbers of PMUs needed to be placed in a PS to make it completely observable. A PS is identified as observable when the voltages of all buses in the system are known. Many algorithms for OPP are developed and presented in the literature which can be broadly classified into two categories: mathematical and heuristic algorithms. A 
comprehensive taxonomy on the basis of these optimization algorithms is presented in [16].

Among many algorithms employed for OPP, integer programming (IP) is the most frequently used strategy. It is a mathematical programming method for solving an optimization problem having integer design variables where the objective function and the constraints are linear, nonlinear, or quadratic. Different researchers presented various formulations for the OPP including constraints like single or multiple branch outage, conventional injection measurements, failure of one or multiple PMUs, the effect of communications lines, etc [16].

OPP problem also addressed specific problems like fault location (FL) observability [17], bad data detection and reduction of errors in state estimation [18, 19] and enhancement on error identification [20]. A hybrid state estimation concept via Gauss-Newton method is introduced in [21]. Simultaneous placement of PMUs and PDCs along with communication infrastructure issues are discussed in [22]. Observability of the network using OPP in both normal condition and islanding condition for active distribution grids is presented in [23] and [24].

\subsubsection{Potential research areas in $O P P$}

Based on the literature review for OPP algorithms, a few potential research areas are provided below:

1) Literature review shows that the OPP problem does not provide a unique solution. Different solutions produce the same minimum number of PMUs at different locations based on the observability criteria and constraints used. Research needs to be done to evaluate the best outcomes based on system constraints and network topology.

2) Optimization strategy should include multiple objectives like installation cost, redundancy, and performance to provide more practical results.

3) Constraints such as communication failure, latency, malfunctioning, noise error, environmental conditions should take into consideration.

4) Majority of the methods presented in the literature works well for small systems. Designing and implementation of robust optimization algorithms applicable to large PS should be investigated.

5) With the advent of micro PMUs ( $\mu$ PMUs) for the distribution system, work needs to be done for OPP for distribution systems especially in the presence of DG.

6) Hybrid methods can be used with the combination of mathematical and heuristic algorithms. Literature review shows great success by combing heuristic methods with integer programming.

\section{Applications of ST in power systems}

\subsection{Applications of PMUs in power transmission systems}

RT grid applications require RT data collection and processing with immediate analysis and visualization. Due to the very high sampling speed, granularity into phase angles and time synchronization, many RT operations are possible using PMUs. PMU data enable excellent visualization, analytics and alarming which can improve operators ability to see and understand the dynamics of bulk PS. It can also help to anticipate or identify potential problems, and evaluate, implement and assess remedial measures. This section reviews RT applications of PMUs in transmission systems.

\subsubsection{Wide area situational awareness and monitoring}

One of the major online applications of PMU data is wide area situational awareness and monitoring. After several cascading outages in the U.S eastern and western interconnection, many utilities developed in-house tools to provide wide-area visualization in order to monitor the dynamics of the grid in real time. These tools provide RT monitoring of grid and also trigger alarms predicting abnormal system conditions. One such example is phasor RT dynamics monitoring system (RTDMS) by Electric Power Group which is a synchrophasor software application for providing RT wide-area situational awareness to operators [25]. It can provide small signal stability monitoring, voltage and frequency stability monitoring, phase angle differences and can also trigger alarms on abnormal system conditions.

Southern California Edison (SCE) also developed a similar type of prototype of a situational awareness tool and named it synchronized measurement and analysis in real time (SMART) tool. An advanced wide-area PS visualization application (WAPSVA) for the RT reliability monitoring was developed by [26] which can be further used as a post-event analysis. This application can show the location, magnitude and the related event message on the visualization display in RT by integration with the online event triggering and location of disturbance applications.

\section{1) Wide area frequency monitoring}

PS frequency is the key indicator of the load-resource balance. For example, the size of a generation loss can be correlated with the size of frequency deviation. Figure 4 shows an example of frequency response to a generation outage at WECC [7]. PMU frequency plot is a good indi- 


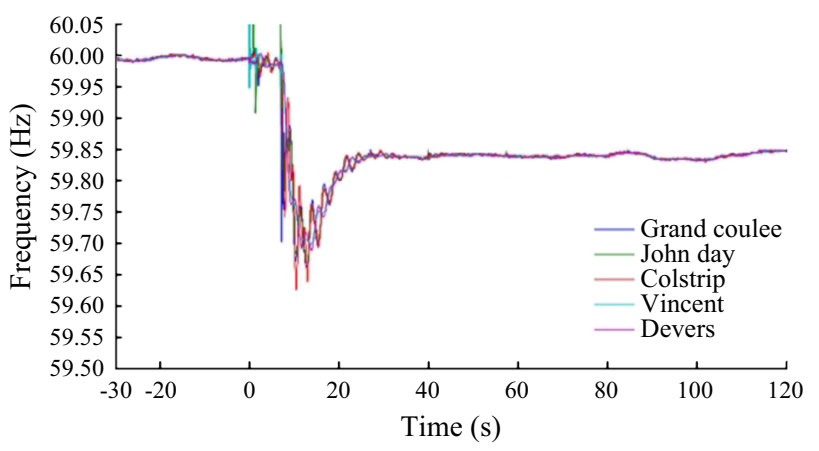

Fig. 4 Western system frequency during a large generation outage on July 17,2002

cator of the lost generation. PMU based wide area frequency monitoring readings can also be used in the postdisturbance analysis. A very good example of wide area frequency monitoring is wide-area frequency monitoring network (FNET) constructed in Virginia tech by utilizing the frequency disturbance recorder (FDR) data coming from the three interconnections in North America [27].

Wide area frequency monitoring applications can be divided into RT applications and non-real time applications depending on their reporting time frame. Non-real time applications are flexible in terms of reporting time frame after receiving the data, however, RT applications require response time in milliseconds. The applications such as event trigger alarming after receiving disturbances from FDR, interarea and local mode oscillation trigger and event visualization are discussed in [28]. A similar type of visualization tool is developed and presented by [29] which shows the RT location, magnitude and the corresponding event message on the frequency display.

\section{2) Voltage stability monitoring}

Voltage stability is the ability of a PS to maintain steady voltages at all buses in the system after being subjected to a disturbance from a given initial operating condition. Different types of voltage stability can be found in [30]. Voltage stability indices (VSI) are used by the operators to know how close the system is to voltage collapse, or how much power can be supplied to loads. PMU data helps to predict voltage instability in real time to perform better control actions in order to avoid cascading outages. Various methods to visualize and analyze the voltage instability condition using RT PMU data are provided in [31-35]. Algorithms to perform voltage stability can be classified into two broader categories: Jacobian matrix based VSI and system variable based VSI [34, 35].

VSI developed in [31] is based on PMU measurements available in WAMSs. This VSI determines the voltage stability margins of all system load buses and correctly predicts voltage collapse as it approaches zero. In [32], an adaptive three-bus power network equivalent is proposed for estimating the voltage stability margin. This approach assumes that synchronized voltage and current phasors are coming from PMUs located on different buses in the PS. A singular value decomposition (SVD) metric to indicate grid vulnerability against voltage instability is proposed in [33]. The presented method for long-term voltage stability requires no information of system parameters or topology information. PMUs are used to get the voltage and current measurements from different buses for approximating a voltage stability indicator in near real time. Some recent PMU based applications for voltage stability are summarized in Table 3 [36-52].

\section{3) Oscillation monitoring and detection}

Oscillations occur in PS primarily due to rotor angle disturbances. Poorly damped or unstable oscillations can lead to undesirable system conditions such as instabilities and cascading outages which can ultimately lead to catastrophic blackouts such as 1996 blackout of the western U.S./Canada interconnected PS. Oscillations in the power grid can be divided into two categories i.e., forced oscillations and electromechanical oscillations [53].

Forced oscillations are usually caused by some rogue input driving the system, rather than a property of the system itself. Whereas, intra-plant mode oscillations, local plant mode oscillations, inter-area mode oscillations, control mode oscillations are all types of electromechanical mode oscillations. PMU data is very helpful to detect undamped electromechanical oscillations due to its high sampling rate. Such type of oscillations can lead up to generator failure. So, asset management can also be performed by implementing control using PS stabilizers and flexible AC transmission system (FACTS) devices using local control modes $[54,55]$. A self-coherence method for detecting sustained oscillations by using PMU data has been presented in [56]. Similarly, a cross-coherence method using multiple-channel PMU data is presented in [57]. Event location such as generator tripping is presented in [58] by triangulating frequency responses. Moreover, a model validation approach to locating the source of oscillation based on post-fault analysis is presented in [59]. An algorithm for the detection and frequency estimation of periodic forced oscillations is proposed in [60].

\subsubsection{State estimation}

State estimation (SE) is a process of estimating the state of the network based on the available measurements. It is not feasible having measurements at every node of the system, therefore, SE is being widely used to estimate the value of state variables where measurement devices are not available. This is an imperative tool for on-line monitoring, 
Table 3 Applications of ST in transmission systems

\begin{tabular}{l} 
Application \\
\hline Coltage stability and monitoring \\
PMU data is used to calculate VSI which indicates the relative closeness of the instability by \\
producing numerical indices. PMU data also helps to predict small disturbance and long \\
disturbance (short term and long term) voltage stability in RT \\
Probabilistic-based voltage stability is proposed using techniques i.e. Monte Carlo simulation, \\
D' matrix and nonlinear optimal power flow \\
VSI using Thevenin equivalent \\
Decision Tree based voltage stability method is presented which requires off-line training to \\
build pre-defined trees and uses PMUs to identify pre-determined voltage stability status \\
Denoising of the PMU data is performed using wavelet shrinkage-based method. Hilbert \\
analysis is used to identify non-linear trends in the denoised data. PMUs based LPF is used to \\
remove anti-aliasing frequency component \\
Limitations of Prony based analysis and Hilbert method is discussed by using stationary and \\
non-stationary signals. Both methods can identify the signals but cannot distinguish two \\
separate modes if the difference in frequency or damping ratio is small \\
A robust least square estimation using ARMAX model is used to detect electromechanical \\
modes. A robust objective function is built to minimize the effect of outliers and data loss \\
from the measurement data \\
Total least square estimation of signal parameters via rotational invariance techniques (TLS- \\
ESPRIT) is introduced to study the effect of colored Gaussian noise on mode estimation \\
Different methods available in model-based approach which includes Prony, matrix pencil, and \\
Hankel total least squares (HTLS) are used to study oscillation monitoring from PMU data. \\
Amount of noise present in the synchrophasor data make it difficult to identify different \\
modes
\end{tabular}

State estimation

Fault location identification and protective relaying
A tracking three-phase state estimator based on modal components of a three-phase system is developed which uses the robust LAV estimation method against intentionally introduced or random bad data. With the help of PMUs, LAV becomes computationally competitive with WLS and eliminates leverage measurements with simple strategic scaling

Phasor only state estimator (PSE) on a large PS using PMU data is developed. A scheme is proposed to detect and isolate transient events using detrended fluctuation analysis which indicates the need to rerun the SE if bad data or transients are present in the measurements

Two different supervisory protection methods are presented. In first, PMU data is used for event identification by forming a supervisory layer which verifies every relay operation and informs the operator about any misoperation. In second, PMU data is analyzed for most prominent disturbance signature. PMU data is fed to the strongest signal selection and fault detection (SSFD) module which preprocesses the signals by removing bad data and identify the data stream with the highest strength

An adaptive PMU based protection scheme for both transposed and untransposed parallel transmission lines which is based on the distributed line model and the synchronized phasor measurements at both ends of lines is discussed

Another adaptive wide area backup protection scheme for transmission lines is proposed in which backup protection zones are formed based on PMU placement and network topology. It also provides a non-iterative solution for the faulted line and FL identification problem analysis, and control of PS. Traditionally, entire PS measurements are obtained through remote terminal units (RTU) of SCADA systems which have both analog and logical measurements. State estimator uses a set of analog measurements such as bus $V, I, P$ and $Q$ for estimating the unknown variables.

PMU based SE was first proposed in 1985 which took the advantage of linear state estimation (LSE). In LSE, voltage variables are expressed in rectangular form and the least squares problem to reduce measurement noise is solved in one step [61]. Applications of LSE using PMU data further extended by [62, 63] to correct for: phase biases in PMU channels; current scaling errors; transformer tap ratios. SE can be categorized into the following three classes: static SE (SSE); tracking SE (TSE); dynamic SE (DSE) [64]. 
Table 4 PMU based SE strategy

\begin{tabular}{ll}
\hline SE strategy & References \\
\hline Static SE & {$[61,62,70]$} \\
Dynamic SE & {$[46,64-67]$} \\
Distributed SE & {$[68,69]$} \\
\hline
\end{tabular}

SSE estimates the PS state associated with a given time and based on the measurement set corresponding to that moment of time. The SSE algorithm uses a flat start for initialization and it is iterative in nature. Therefore, it is computationally heavy and cannot be executed in small intervals. To overcome this problem, TSE was introduced where the estimation starts from the last calculated state variables instead of a flat point. In both types, the PS state is estimated based on a single set of measurements [65]. However, in the DSE, the system states are continuously monitored at the regular interval. It is becoming more prevalent in the modern energy management systems due to its ability to predict the PS state progressively in short time steps. Each time step of DSE, both measurement and prediction data sets are used. A comprehensive survey of DSE techniques and model implementation using PMU data are presented in [66, 67]. Moreover, Table 4 provides some references categorized by SE based strategies using synchrophasor measurements [46, 61, 62, 64-71].

The computational procedure involved in $\mathrm{SE}$ is an optimization function, which can be first order weighted least squares (WLS), linear programming based on least absolute value (LAV) estimator or second order of the derivative which involves the Lagrangian Hessian matrix. However, the common method used to solve the static SE is WLS and LAV. PMU based SE adds the advantage of the non-iterative process and the linearity of the system by using all the above methods [71]. Table 3 also summarizes some PMU based state estimation applications.

\subsubsection{Fault location, identification and protective relaying}

PMU based time-synchronized measurements are also used in relay protection and FL identification. PS Relaying Committee Report of Working Group C-14 presented some of the useful relaying applications which cover power swing detection, line reclosing selectivity, synchrophasor assisted black start, automatic generator shedding, distance protection, bus differential protection and line differential protection [72].

Out of step protection is important for large systems with a huge number of machines in order to avoid a system collapse. Conventional out of step protection schemes are usually based on positive sequence impedance at the relay location. PMUs provide an added advantage of phase angle measurements which can be useful in designing out of step protection by adding fast communication systems. Different schemes for out-of-step protection by taking advantage of phase angle differences using synchrophasor measurements are presented in [73, 74]. These schemes include detecting out-of-step protection based on RT swing curves and RT extended equal area criterion. The concept of adaptive out-of-step protection is implemented at the Florida-Georgia interface using synchrophasor measurements [75]. Tokyo Electric Power Company (TEPCO, Japan) implemented a predictive out-of-step protection system based on phase differences between power centers using the RT phasor measurement principle [76]. A combined out-of-step prediction and relay blocking scheme is presented in [77]. This scheme uses line end PMU inputs for detecting vulnerable relays and blocking their zone 1 trip for a limited time interval before ingression into zone 1 .

PMUs also play a major role in developing adaptive protection schemes as it provides fast communication. An adaptive relay is a protection device whose characteristics can be altered on the basis of external information. Some adaptive protection applications using PMU data are summarized in Table 3. Similarly, event detection and identification using PMU data can play an important role in verifying a relay operation which is the principle of supervisory protection. Two cases of supervisory protection using PMU data are also presented in Table 3. Different FL/detection techniques for transmission lines using synchronized phasor measurements are presented in [78]. FL schemes for a combined overhead line with underground power cable are presented in [79]. Another FL technique for a two-terminal multisection compound transmission line using synchrophasor measurements is presented and successfully implemented in Taiwan, China [80].

\subsection{Potential research areas for ST applications in transmission systems}

Wide area visualization is becoming a reality for transmission system operators. Through situational awareness, the control system responsible for monitoring critical infrastructure and SCADA system can detect malicious incidents and threats on the electric grid. However, research needs to be done in order to develop commercial computational tools and establishing processes that can effectively use PMU data. Similarly, mapping of a huge amount of data from ST can be used to address event analysis and control actions. Another potential research area is data analysis of post-disturbance data coming from PMUs. This data can be used to build potential machine 
learning algorithms using pattern recognition and pattern matching techniques.

ST applications for power system protection mainly focuses on FL, fault detection and fault isolation with ST data from one or both ends of the transmission lines. Use of PMU data with conventional traveling-wave and impedance-based method leads to a better performance in system protection. Moreover, the operation of the PMU based protection schemes is more flexible than the traditional protection schemes. Some future applications in PSP using PMU data includes the implementation of knowledgebased methods which uses computer programming to solve complex problems. Common examples of knowledgebased methods that can be used for PSP are artificial neural networks (ANNs), support-vector machines (SVM), signal processing, and data matching etc.

Another potential future application of ST lies in the area of asset management. With the widespread deployment of PMUs in U.S. grid, many grid operators and transmission owners are in the opinion that they can use the PMU data to gain unprecedented visibility into the status and health of PS equipment. A comprehensive report on how the behavior of generation and transmission assets like generators, power transformers, and measurement transformers can be predicted is presented in [81]. This report also discussed the methods of calculating the life expectancy of these assets by using the granulated PMU data. Different preventive techniques can be applied to these assets to enhance their power quality and life.

\subsection{Applications of PMUs in power distribution systems}

In comparison to transmission systems, distribution systems have reduced line lengths and limited power flows. This results in very small phase differences between voltage phasors of different buses. Hence, PMUs can play an important role in monitoring, control, and protection of distribution systems. Previously, utility engineers were only interested in the magnitudes of voltages because of their small value but now because of dynamic load changing due to distributed energy resources (DER), the phase angle is also playing an important part in the analysis of distribution systems. Therefore, ST is gaining importance in the distribution systems and it will only increase with the addition of more DER.

Significant work has been already carried out in order to apply ST in distribution systems. For example, $\mu \mathrm{PMU}$, which provides RT synchrophasor data from the consumer voltage can play a huge role in re-shaping the distribution grid. They are much cheaper than current commercial PMUs. Some PMU based distribution system are presented next.

\subsubsection{Islanding detection}

Islanding refers to the condition in which a distributed generation (DG) continues to power a location even in the absence of power from the electric utility. Two types of islanding can occur in a PS: intentional and unintentional. Intentional islanding is used for maintenance of electric grid and unintentional islanding occurs due to equipment failure or fault conditions [82]. Islanding introduces threats to utility workers during and mis-coordination of protective devices. Due to this reason, DG must detect islanding and immediately stop supplying power to the islanded network. IEEE Standard 1547-2008 states that the DG must be disconnected from the isolated grid within $2 \mathrm{~s}$ after an unintentional islanding event [83]. PMUs can be very helpful to detect islanding conditions and with the help of fast and secure communication, better control actions can be performed within $2 \mathrm{~s}$ to disconnect the DG from the grid.

Initially, synchrophasor based islanding detection schemes were proposed which were based on offline simulations and did not include the RT data from PMUs. These schemes were unable to predict the communication latencies because of their offline modeling [84, 85]. Methods for islanding detection in a distributed system with DG can be classified into three categories: active methods which include active frequency drifting method and phase-shift method, passive methods that include over/under voltage, over/under frequency and communication-based methods like power-line-signaling-based scheme [86-88]. On basis of these, two islanding detection methods; frequency difference and change of angle difference method for DG, are presented in [89] by using the data coming from a PMU based FDR and different case studies were presented.

The concept of islanding maneuvering of an active distribution network (ADN) using PMU data is presented in [90]. In ADN embedded generation is actively used by the energy management system in order to achieve the operational objectives. The developed PMU is based on a discrete Fourier transform (DFT) tone-reconstruction algorithm by aiming a very low value of TVE as required for distribution applications. The experimental tests were successfully implemented in islanding detection of $80 \mathrm{MW}$ combined cycle power plant (CCPP). An anti-islanding detector is presented in [91] by using PMU measurements communicating through a robust and secure IPv6 telecom network to improve sensitivity and prevent cascading tripping. It also operates in case of failure of an incumbent anti-islanding detector. An online strategy for detecting and estimating PS unintentional islanding using RT PMU data is presented in [92].

A successful implementation of islanding detection with microprocessor relays having PMU capabilities is 
implemented in International Drive, Orlando as shown in Fig. 5. In such a complex system, it would be difficult to determine which combination of open breakers and switchgear would cause a generator out on the distribution system to be islanded with nearby connected loads. But microprocessor-based relays with PMU capabilities made it possible to provide islanding detection at any location in such a complex distribution system. According to IEEE 1547, DG must disconnect for an islanding condition in $2 \mathrm{~s}$ or less, regardless of the load generation balance [93]. It can be seen in [94], that how generator protection, infinite impulse response (IIR) or phase shift, and local islanding detection logic (IDL) schemes are taking a very long operating time as load to generation ratio approaches to 1 , as compared to wide-area scheme which is a combination of phase angle slip and acceleration between the generator location and the remote station location [94].
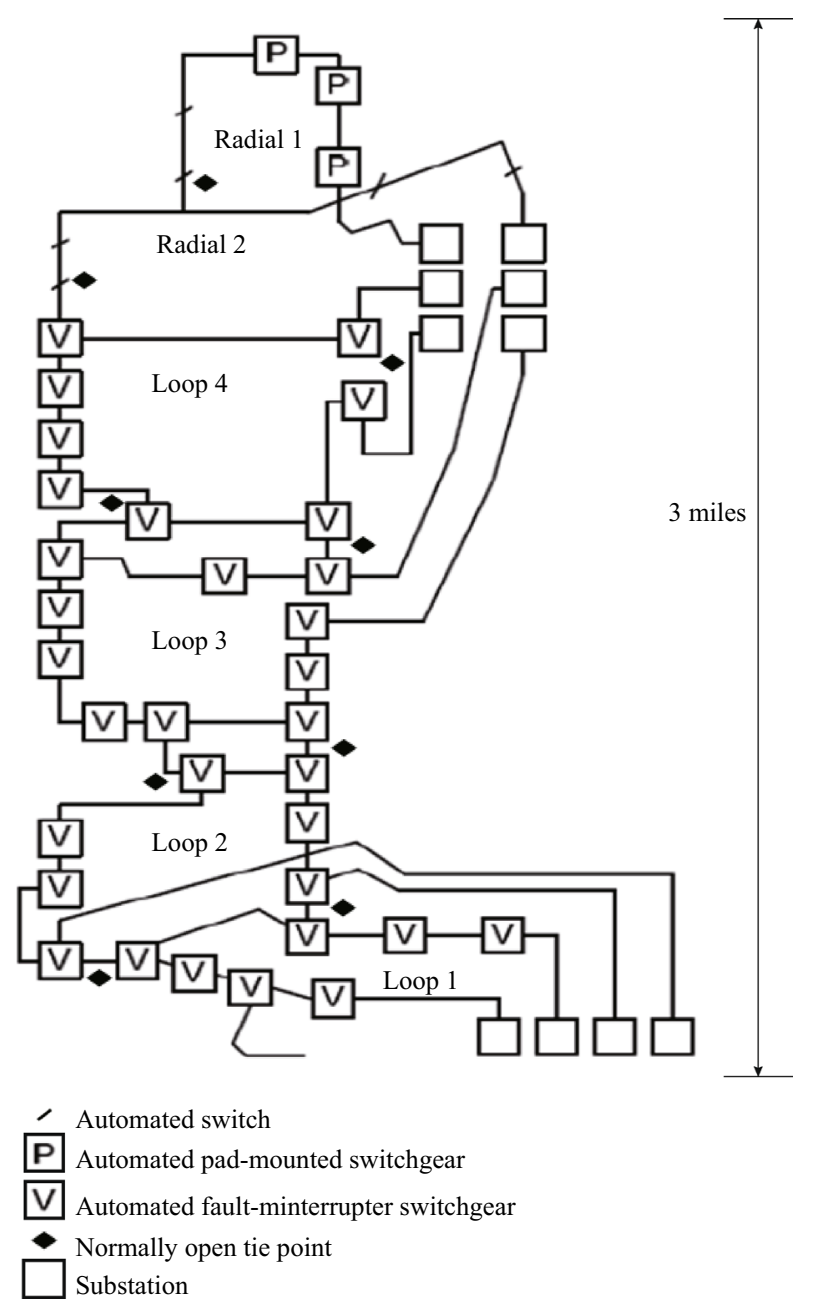

Fig. 5 International drive feeder diagram

\subsubsection{Fault location}

Penetration of DER in distribution systems makes them more complex, unbalanced and vulnerable to faults. Traditionally, FL in distribution network was performed by fault current based methods, impedance-based methods, traveling wave-based methods and signal injection techniques but they all have limitations as explained in [95]. PMUs can be very helpful in locating and detecting the faults in the distribution system because of their high speed and time synchronized phasor measurements.

A FL method using synchrophasors in emerging distribution systems is presented in [96]. This method leverages PMU measurements from multiple locations to pinpoint the faulted line. This is a two-step method in which the first step is to find out the candidate locations at one terminal by iterating every line segment using PMUs. The second step is to identify the actual FL by comparing the voltage phasors for the junction nodes of branches which are calculated using synchrophasors measured from two terminals. For validation, this method is also implemented in a modern substation located in Duke Energy territory and the results were within $1 \%$ accuracy of the line length.

A PMU based FL identification in smart distribution networks is presented in [95] by implementing a state estimation algorithm to identify the FL. Offline models are developed to compare the results by injecting several types of faults in the system. Another SE based FL and identification technique is presented in [97] by computing parallel synchrophasor-based state estimators. The faulted line identified by using LSE values with a latency range of 102-122 ms. A technique for FL based on the comparison of voltage differences is presented in [98] in which FL in a DG based distribution system is performed by calculating the actual pre and post-fault voltage variations and then comparing it with a voltage variation in a hypothetical faulted bus. Instead of using protective devices, PMUs are used for measurement of voltages and currents to calculate thevenin equivalent for sequence impedances. By using a three-phase impedance matrix at the substation bus, FL was calculated based on voltage differences in the circuit.

FL method for low fault currents is presented in [99] in which PMU based current phasors are used because of their sensitivity to detect a small change in current. This technique is very useful for high impedance faults where overcurrent protection is difficult to apply. Aging power cables in both overhead and underground systems pose complexity in detecting the fault with high accuracy. To overcome this problem, a synchrophasor based FL technique is presented in [100]. In this method, PMUs are placed at both ends of the line and then by using distributed line model and modal transformation theory, a fault can be detected. The method was implemented by performing 
simulations in EMTP platform and showed greater accuracy.

\subsubsection{State estimation for distribution systems}

In modern power grids, distribution systems are operated through specifically designed distribution management systems (DMS) which apply control strategies using accurate knowledge of network states. In transmission systems, accurate knowledge of states can be obtained by a numerous number of measuring devices but in a distribution system, measuring devices are not large in number. Therefore, ST data can provide RT SE in distribution systems. Distribution system state estimation (DSSE) is very sensitive because, in a microgrid environment, the communication window between different components is very small; so quick, reliable and secure data needs to be collected in order to perform correct actions downstream and PMU data can provide that [101].

In general, there are two mathematical approaches to include synchronized measurements in state estimation [102]. In the first approach, the estimator design is based on the combined measurements from PMUs and from other conventional measurement devices. This approach results in a formation of a non-linear state estimator. The second approach deals with incorporating the phasor measurements and the results of the traditional SE in a post-processing linear estimator. The linear estimator can be implemented in DMS due to its non-iteration convergence. The first approach is implemented by [103] in which the use of rectangular components of the voltage and current synchrophasors, leads to a linear state estimation. A multiphase power flow model used for state estimation is also presented in this paper. Design of DSSE by using both of these approaches are presented in [104] and [105].

A different method of linear SE in a microgrid environment is presented in [106] which is known as RT SE. A factor graph approach is considered for the bus/branch model and the state variables are divided into two layers: behavior layer and observational layer. A new SE algorithms belief propagation (BP) is presented which deals with sparse measurements based on the initialization of states and refine the estimates with more incoming data. This method also shows that PMU data can increase the accuracy of the estimation. Impact of model uncertainties on the parameter and state estimations are studied in [107] and [108] and proposed the reduction of these effects using PMU measurements.

Although RT data based SE (RTSE) using PMUs is very helpful but its accuracy is highly dependable on the communication infrastructure in a distribution atmosphere. To avoid communication delays and bandwidth problems in an ADN, a communication platform called (C-DAX) is presented in [109]. It offers both quantitative (latency and bandwidth) and qualitative (flexibility, scalability, and resilience) benefits.

\subsubsection{Harmonic estimation}

Large penetration of photovoltaic (PV) in the distribution system brings power quality problems like harmonics from different PV inverters. These harmonics need to be correctly identified in order to maintain power quality to residential and industrial customers [110]. A technique for measurement of harmonic synchrophasors in a distribution system is presented in [111]. In this technique, high-accuracy GPS receivers and general-purpose acquisition hardware are used for measurement purposes. Main causes of uncertainty in the evaluation of synchronized harmonic phasors are also presented. Tests are performed on a threephase low-voltage distribution network and the results to evaluate harmonic synchrophasors using a PMU prototype are within acceptable limits. More applications of synchrophasors in harmonic estimation can be found in [112].

\subsubsection{Load modeling and load characterization}

One of the major concerns related to DG is the impact on system stability due to the interaction between generators and load characteristics. Moreover, load dynamics are also changing due to the increase in a number of electric vehicles and inverter-based loads. Hence, accurate load modeling is required for system operations and analysis. PMU data is very helpful for estimation of distribution network load model. Different algorithms for accurate load modeling using ST in a distribution network are presented in [113] and [114]. One such example is a harmonic search algorithm which works for both normal and transient conditions [113]. In this algorithm, the conventional distribution system is represented by load models in symmetrical parameters that can be paired with symmetrical components of PMU based $V$ and $I$. Whereas, in [114], data from PMUs is used to identify the parameters of composite load modeling. This study shows that the composite load model has a higher accuracy than traditional static load modeling. Another implementation of load modeling of a distribution system using synchrophasor data is performed in Australia which shows that how PMU data combined with rapid load variation identifies new system dynamic [115].

\subsection{Micro PMUs}

Along with more distributed control and smart devices in the distribution grid, $\mu$ PMUs will play an important role to provide new insight into modern PS. These units can be created more cheaply than the current commercial PMUs. 
$\mu$ PMU was initially developed by power sensors limited (PSL) with the University of California and Lawrence Berkeley National Lab (LBNL) in 2015 [116]. The purpose was to address the need for tools to better observe, understand and manage the grid at the distribution scale. This $\mu$ PMU was initially used for diagnostic applications and control applications of the distribution grid. A comprehensive review of the development of $\mu \mathrm{PMU}$ is presented in [117].

A commercial $\mu \mathrm{PMU}$ was developed by [118] in which the main function of the device was to calculate voltage, frequency, and phase at the household voltage level. This unit operates at a consumer level voltage and has the capability to capture only one phase. More details about this device can be found in [118].

\subsection{Potential research areas for ST applications in distribution systems}

With the penetration of more DER, ST is gaining importance in the distribution system. Based on the summary provided in Section 4.3, a number of new potential research areas are presented below:

1) Shorter line lengths, smaller power angles, and higher harmonic levels are some of the key differentiating factors that distribution system presents for ST. Hence, it is important to revisit the typical measurement error of the PMU. Also, different applications have different vulnerability levels towards measurement errors that can be evaluated prior to the usage of PMU data.

2) The proliferation of intelligent electronic devices (IEDs) in the smart grid makes it more intelligent by enabling two-way communication between the grid and the customer. However, it makes the distribution system more vulnerable to external threats and cybersecurity problems. Similarly, if one PMU is compromised by an attacker, they can threaten the security at large-scale. A multi-layer architecture protected by security gateways needs to be created in case of compromising PMU data.

3) With the increasing number of electric vehicles (EVs) due to climate change policies, it is envisioned that they may be used to power the electric grid in the future. Vehicle to grid (V2G) service can be used to provide services like peak load shaving, smoothing generation from non-dispatchable renewable energy resources and may act as a reserve against unexpected outages [119, 120]. Additionally, by the deployment of fast responding frequency measuring devices like PMU, EVs can be used in primary reserve service.

4) With increasing penetration of DER in the grid, new protection schemes can be developed using ST. Smart measurement devices and sensors can help to automate system protection that can lead to a self-healing smart grid.

5) Development of advanced data analytic models for event-driven data and machine learning algorithms can be incorporated in the existing methodologies.

\section{Conclusion}

This paper provides an in-depth literature review of ST, its architecture, placement techniques and applications in power transmission and distribution systems. The review also presents potential research areas for OPP techniques and ST applications in transmission and distribution systems. PMUs are deployed in large number in transmission networks but with the addition of more DG resources and the advent of $\mu$ PMUs, they are becoming popular in the distribution system. Most of the applications presented in this paper are currently in practice in the electric power industry. Some evolving applications also exist in academic publications and will be part of the electric power industry in the near future. These applications use data analytics and machine learning algorithms for power systems security and resilience. With ST, the benefits gained in terms of reliability, resiliency and operational efficiency of the grid justifies its installment. It is expected that with further proliferation of data resources in the power grid, ST can perform many of the aforementioned tasks with more accuracy and reliability.

Open Access This article is distributed under the terms of the Creative Commons Attribution 4.0 International License (http:// creativecommons.org/licenses/by/4.0/), which permits unrestricted use, distribution, and reproduction in any medium, provided you give appropriate credit to the original author(s) and the source, provide a link to the Creative Commons license, and indicate if changes were made.

\section{References}

[1] Saadat H (2002) Power systems analysis, 2nd edn. PSA Publishing LLC, Portland

[2] Kosterev N, Taylor W, Mittelstadt A (1999) Model validation for the August 10, 1996 WSCC system outage. IEEE Trans Power Syst 14(3):967-979

[3] Baldick R, Chowdhury B, Dobson I et al (2008) Initial review of methods for cascading failure analysis in electric power transmission systems IEEE PES CAMS task force on understanding, prediction, mitigation and restoration of cascading failures. In: Proceedings of IEEE PES general meeting, Pittsburgh, USA, 20-24 July 2008, 8 pp

[4] Dagle JE (2010) The North American SynchroPhasor Initiative (NASPI). In: Proceedings of IEEE PES general meeting, Providence, USA, 25-29 July 2010, 3 pp 
[5] Hurtgen M, Maun C (2012) Applications of PMU measurements in the Belgian electrical grid. Tech Rep: 1-75

[6] Phadke G, Thorp J (2017) Synchronized phasor measurements and their applications, 2nd edn. Springer, USA

[7] NERC (2010) Real-time application of synchrophasors for improving reliability 2010. https://www.smartgrid.gov/files/ RAPIR_final_101710.pdf. Accessed 20 April 2016

[8] IEEE (2011) IEEE C37.118.1-2011: IEEE standard for synchrophasor measurements for power systems

[9] IEEE (2014) IEEE C37.118.1a-2014: IEEE standard for synchrophasor measurements for power systems

[10] Gurusinghe R, Rajapakse D, Muthumuni D (2013) Modeling of a synchrophasor measurement unit in an electromagnetic transient simulation program. In: Proceedings of the 2013 international conference on power system transients, Vancouver, Canada, 2013, 6 pp

[11] Silverstein A (2017) Synchrophasors and the grid. https://www. naspi.org/sites/default/files/reference_documents/naspi_naruc_ silverstein_20170714.pdf. Accessed 21 March 2017

[12] CERTS. http://www.phasor-rtdms.com/. Accessed 23 March 2017

[13] IEEE (2013) IEEE C37.244-2013 Phasor data concentrator requirements for power system protection, control, and monitoring

[14] Martin K, Chair W, Brunello G et al (2014) An overview of the IEEE standard C37.118.2 synchrophasor data transfer for power systems. IEEE Trans Smart Grid 5(4):1980-1984

[15] Baldwin T, Mili L, Boisen M (1993) Power system observability with minimal phasor measurement placement. IEEE Trans Power Syst 8(2):707-715

[16] Manousakis N, Korres N, Georgilakis P (2012) Taxonomy of PMU placement methodologies. IEEE Trans Power Syst 27(2):1070-1077

[17] Lein KP, Liu CW, Yu CS (2006) Transmission network fault location observability with minimal PMU placement. IEEE Trans Power Del 21(3):1128-1136

[18] Gou B, Kavasseri RG (2014) United PMU placement for observability and bad data detection in state estimation. IEEE Trans Power Syst 29(6):2573-2580

[19] Zhang L, Abur A (2013) Strategic placement of phasor measurements for parameter error identification. IEEE Trans Power Syst 28(1):393-400

[20] Zhang L, Abur A (2012) Single and double edge cutset identification in large scale power networks. IEEE Trans Power Syst 27(1):510-516

[21] Li X, Scaglione A, Chang TH et al (2014) A framework for phasor measurement placement in hybrid state estimation via Gauss Newton. IEEE Trans Power Syst 29(2):824-832

[22] Fesharaki FH, Hooshmand RA, Khodabakhshian A et al (2014) Simultaneous optimal design of measurement and communication infrastructures in hierarchical structured WAMS. IEEE Trans Smart Grid 5(1):312-319

[23] Huang L, Sun Y, Xu J et al (2014) Optimal PMU placement considering controlled islanding of power system. IEEE Trans Power Syst 29(2):742-755

[24] Liu J, Ponci F, Monti A et al (2014) Optimal meter placement for robust measurement systems in active distribution grids. IEEE Trans Instrum Meas 63(5):1096-1105

[25] http://www.electricpowergroup.com/rtdms.html. Accessed 21 April 2016

[26] Bhargava B, Salazar A (2008) Synchronized measurement and analysis in real-time SMART system at Southern California Edison (SCE) Co. In: Proceedings of IEEE/PES transmission and distribution conference and exposition: Latin America, Bogota, Colombia, 13-15 August 2008, 6 pp
[27] Zhou M, Centeno A, Thorp J (2006) An alternative for including phasor measurements in state estimators. IEEE Trans Power Syst 21(4):1930-1937

[28] Zhong Z, Xu C, Billian B (2005) Power system frequency monitoring network (FNET) implementation. IEEE Trans Power Syst 20(4):1914-1921

[29] Zhang Y, Markham P, Xia T (2010) Wide-area frequency monitoring network (FNET) architecture and applications. IEEE Trans Smart Grid 1(2):159-167

[30] Kundur P, Paserba J, Ajjarapu V (2004) Definition and classification of power system stability IEEE/CIGRE joint task force on stability terms and definitions. IEEE Trans Power Syst 9(3):1387-1401

[31] Gong Y, Schulz N, Guzman A (2006) Synchrophasor-based real-time voltage stability index. In: Proceedings of IEEE PES power systems conference and exposition, Atlanta, USA, 29 October-1 November 2006, 8 pp

[32] Hu F, Sun K, Rosso A et al (2014) An adaptive three-bus power system equivalent for estimating voltage stability margin from synchronized phasor measurements. In: Proceedings of IEEE PES general meeting, National Harbor, USA, 27-31 July 2014, $5 \mathrm{pp}$

[33] Lim J, DeMarco C (2016) SVD-based voltage stability assessment from phasor measurement unit data. IEEE Trans Power Syst 21(4):2557-2565

[34] Karbalaei F, Soleymani H, Afsharnia S (2010) A comparison of voltage collapse proximity indicators. In: Proceedings of the IPEC conference, Singapore, 27-29 October 2010, 4 pp

[35] Cupelli M, Cardet C, Monti A (2012) Voltage stability indices comparison on the IEEE-39 bus system using RTDS. In: Proceedings of IEEE international conference on power system technology (POWERCON), Auckland, New Zealand, 30 October-2 November 2012, 6 pp

[36] Pulok M, Faruque M (2015) Utilization of PMU data to evaluate the effectiveness of voltage stability boundary and indices. In: Proceedings of North American power symposium, Charlotte, USA, 4-6 October 2015, 6 pp

[37] Rodrigues A, Prada R, Silva M (2010) Voltage stability probabilistic assessment in composite systems: modeling unsolvability and controllability loss. IEEE Trans Power Syst 25(3): 1575-1588

[38] Liu H, Chu C (2014) Wide-area measurement-based voltage stability indicators by modified coupled single-port models. IEEE Trans Power Syst 29(2):756-764

[39] Gao Q, Rovnyak S (2011) Decision trees using synchronized phasor measurements for wide-area response-based control. IEEE Trans Power Syst 26(2):855-861

[40] Teeuwsen SP, Erlich I, El-Sharkawi MA et al (2006) Genetic algorithm and decision tree-based oscillatory stability assessment. IEEE Trans Power Syst 21(2):746-753

[41] Messina AR, Vittal V, Heydt GT (2009) Nonstationary approaches to trend identification and denoising of measured power system oscillations. IEEE Trans Power Syst 24(4):1798-1807

[42] Browne TJ, Vittal V, Heydt GT (2008) A comparative assessment of two techniques for modal identification from power system measurements. IEEE Trans Power Syst 23(3):1408-1415

[43] Zhou N, Trudnowski DJ, Pierre JW (2008) Electromechanical mode online estimation using regularized robust RLS methods. IEEE Trans Power Syst 23(4):1670-1680

[44] Tripathy P, Srivastava S, Singh S (2011) A modified TLSESPRIT based method for low-frequency mode identification in power systems utilizing synchrophasor measurements. IEEE Trans Power Syst 26(2):719-727 
[45] Liu G, Quintero J, Venkatasubramanian V (2007) Oscillation monitoring system based on wide area synchrophasors in power systems. In: Proceedings of iREP symposium on bulk power system dynamics and control, Charleston, USA, 19-24 August 2007, $13 \mathrm{pp}$

[46] Gol M, Abur A (2014) A Robust PMU based three-phase state estimator using modal decoupling. IEEE Trans Power Syst 29(5):2292-2299

[47] Ashton P, Taylor G, Irving M et al (2013) Novel application of detrended fluctuation analysis for state estimation using synchrophasor measurements. IEEE Trans Power Syst 28(2):1930-1938

[48] Dahal O, Cao H, Brahma S et al (2014) Evaluating performance of classifiers for supervisory protection using disturbance data from phasor measurement units. In: Proceedings of IEEE PES innovative smart grid technologies, Istanbul, Turkey, 12-15 October 2014, 6 pp

[49] Biswal M, Brahma S, Cao H (2016) Supervisory protection and automated event diagnosis using PMU data. IEEE Trans Power Del 31(4):1855-1863

[50] Chen C, Liu C, Jiang J (2002) A new adaptive PMU based protection scheme for transposed/untransposed parallel transmission lines. IEEE Trans Power Del 17(2):395-404

[51] Lin Y, Liu C, Chen C (2014) An adaptive PMU based fault detection/location technique for transmission lines with consideration of arcing fault discrimination Part II : performance evaluation. IEEE Trans Power Del 19(4):1594-1601

[52] Neyestanaki M, Ranjbar A (2015) An adaptive PMU-based wide area backup protection scheme for power transmission lines. IEEE Trans Smart Grid 6(3):1550-1559

[53] Follum J, Pierre J (2016) Detection of periodic forced oscillations in power systems. IEEE Trans Power Syst 31(3):2423-2433

[54] Messina A, Vittal V (2006) Nonlinear, non-stationary analysis of inter-area oscillations via hilbert spectral analysis. IEEE Trans Power Syst 21(3):1234-1241

[55] Kakimoto N, Sugumi M, Makino T et al (2006) Monitoring of inter-area oscillation mode by synchronized phasor measurement. IEEE Trans Power Syst 21(1):260-268

[56] Zhou N, Dagle J (2015) Initial results in using a self-coherence method for detecting sustained oscillations. In: Proceedings of IEEE PES general meeting, Denver, USA, 26-30 July 2015, 1 $\mathrm{pp}$

[57] Zhou N (2016) A cross-coherence method for detecting oscillations. IEEE Trans Power Syst 31(1):623-631

[58] Gardner RM, Wang JK, Liu Y (2006) Power system event location analysis using wide-area measurements. In: Proceedings of IEEE PES general meeting, Montreal, Canada, 18-22 June 2006, 7 pp

[59] Ma J, Zhang P, Fu H (2010) Application of phasor measurement unit on locating disturbance source for low-frequency oscillation. IEEE Trans Smart Grid 1(3):340-346

[60] Follum J, Pierre J (2017) Simultaneous estimation of electromechanical modes and forced oscillations. IEEE Trans Power Syst 32(5):3958-3967

[61] Thorp J, Phadke A, Karimi K (1985) Real-time voltage phasor measurements for static state estimation. IEEE Trans Power Appar Syst 89(11):3098-3106

[62] Vanfretti L, Chow J, Sarawgi S (2011) A phasor data based state estimator incorporating phase bias correction. IEEE Trans Power Syst 26(1):111-119

[63] Ghiocel S, Chow J, Stefopoulos G (2011) Phasor measurement based state estimation for synchrophasor data quality improvement and power transfer interface monitoring. IEEE Trans Power Syst 29(2):881-888
[64] Silva A, Filho M, Cantera J (1987) An efficient dynamic state estimation algorithm including bad data processin. IEEE Power Eng Rev 7(11):1050-1058

[65] Aminifar F, Shahidehpour M, Firuzabad M (2014) Power system dynamic state estimation with synchronized phasor measurements. IEEE Trans Instrum Meas 63(2):352-363

[66] Filho M, Souza J (2009) Forecasting aided state estimation Part I: panorama. IEEE Trans Power Syst 24(4):1667-1677

[67] Filho M, Souza J, Freund R (2009) Forecasting-aided state estimation Part II: implementation. IEEE Trans Power Syst 24(4):1678-1685

[68] Jiang W, Vittal V, Heydt T (2007) A distributed state estimator utilizing synchronized phasor measurements. IEEE Trans Power Syst 22(2):563-571

[69] Jiang W, Vittal V, Heydt T (2008) Diakoptic state estimation using phasor measurement units. IEEE Trans Power Syst 23(4):1580-1589

[70] Bi T, Qin X, Yang Q (2008) A novel hybrid state estimator for including synchronized phasor measurements. Electr Power Syst Res 78(8):1343-1352

[71] Pires R, Costa A, Mili L (1999) Iteratively reweighted least squares state estimation through givens rotation. IEEE Trans Power Syst 14(4):1499-1507

[72] O'Brien J, Deronja A, Apostolov A et al (2014) Use of synchrophasor measurements in protective relaying applications. In: Proceedings of the 67th annual conference for protective relay engineers, College Station, USA, 31 March-3 April 2014, $7 \mathrm{pp}$

[73] Centeno V, Phadke A, Edris A (1997) An adaptive out-of-step relay for power system protection. IEEE Trans Power Del 12(1):61-71

[74] Thorp J, Phadke A, Horowitz S et al (1988) Some applications of phasor measurements to adaptive protection. IEEE Trans Power Syst 3(2):791-798

[75] Centeno V, Ree J, Phadke A (1993) Adaptive out-of-step relaying with phasor measurement techniques. IEEE Comput Appl Power 6(4):12-17

[76] Ohura Y, Suzuki M, Yanagihashi K (1990) A predictive out-ofstep protection system based on observation of the phase difference between substations. IEEE Trans Power Del 5(4):1695-1704

[77] Lavand S, Soman S (2016) Predictive analytic to supervise zone 1 of distance relay using synchrophasors. IEEE Trans Power Del 31(4):1844-1854

[78] Liu C, Lien k, Chen C et al (2008) A universal fault location technique for $\mathrm{N}$-terminal $(\mathrm{N}-3)$ transmission lines. IEEE Trans Power Del 23(3):1366-1373

[79] Yang X, Choi M, Lee S et al (2008) Fault location for underground power cable using distributed parameter approach. IEEE Trans Power Syst 23(4):1809-1816

[80] Liu C, Lin T, Yu C (2012) A fault location technique for twoterminal multisection compound transmission lines using synchronized phasor measurements. IEEE Trans Smart Grid 3(1):113-121

[81] NASPI (2015) Technical report on diagnosing equipment health and mis-operations with PMU data

[82] Almas M, Vanfretti L (2016) RT-HIL implementation of hybrid synchrophasor and GOOSE-based passive islanding schemes. IEEE Trans Power Del 31(3):1299-1309

[83] IEEE (2009) IEEE standard for interconnecting distributed resources with electric power systems. IEEE Std 1547.2:2008

[84] Franco R, Sena C, Taranto GN (2013) Using synchro-phasors for controlled islanding - a prospective application for the Uruguayan power system. IEEE Trans Power Syst 28(2):2016-2024 
[85] Laverty D, Best D, Morrow D (2015) Loss-of-mains protection system by application of phasor measurement unit technology with experimentally assessed threshold settings. IET Gener Transm Distrib 9(2):146-153

[86] Lopes LAC, Sun H (2006) Performance assessment of active frequency drifting islanding detection methods. IEEE Trans Energy Convers 21(1):171-180

[87] Wang WC, Kliber J, Xu W (2009) A scalable power-line-signaling-based scheme for islanding detection of distributed generators. IEEE Trans Power Del 24(2):903-909

[88] Wang X, Freitas W, Xu W (2011) Dynamic non-detection zones of positive feedback anti-islanding methods for inverterbased distributed generators. IEEE Trans Power Del 26(2):1145-1155

[89] Lin Z, Xia T, Ye Y et al (2013) Application of wide area measurement systems to islanding detection of bulk power systems. IEEE Trans Power Syst 28(2):2006-2015

[90] Borghetti A, Nucci C, Paolone M et al (2011) Synchronized phasors monitoring during the islanding maneuver of an active distribution network. IEEE Trans Smart Grid 2(1):82-91

[91] Laverty D, Morrow D, Best R et al (2011) Anti-islanding detection using synchrophasors and internet protocol telecommunications. In: Proceedings of 2nd IEEE PES international conference and exhibition on innovative smart grid technologies, Manchester, UK, 5-7 December 2011, 5 pp

[92] Sun R, Centeno V (2014) Wide area system islanding contingency detecting and warning scheme. IEEE Trans Power Syst 29(6):2581-2589

[93] Basso T (2014) IEEE 1547 and 2030 standards for distributed energy resources interconnection and interoperability with the electricity grid. NREL/TP-5D00-63157

[94] Hataway G, Flerchinger B, Moxley R (2013) Synchrophasors for distribution applications. In: Proceedings of power and energy automation conference, Spokane, USA, 26-28 March 2013, 6 pp

[95] Cordova J, Faruque MO (2015) Fault location identification in smart distribution networks with DG. In: Proceedings of North American power symposium, Charlotte, USA, 4-6 October 2015, 7 pp

[96] Ren J, Venkata S, Sortomme E (2014) An accurate synchrophasor based fault location method for emerging distribution systems. IEEE Trans Power Del 29(1):297-298

[97] Pignati M, Zanni L, Romano P et al (2017) Fault detection and faulted line identification in active distribution networks using synchrophasors-based real-time state estimation. IEEE Trans Power Del 32(1):381-392

[98] Brahma S (2011) Fault location in power distribution system with penetration of DG. IEEE Trans Power Del 26(3):1545-1553

[99] Zanjani MGM, Kargar HK, Zanjani MGM (2012) High impedance fault detection of distribution network by phasor measurement units. In: Proceedings of 17th conference on electrical power distribution networks, Tehran, Iran, 2-3 May 2012, 5 pp

[100] Din E, Gilany M, Aziz M et al (2005) A PMU double ended fault location scheme for aged power cables. In: Proceedings of IEEE PES general meeting, San Francisco, USA, 16 June 2005, $7 \mathrm{pp}$

[101] Abur A (2004) Power system state estimation: theory and implementation. CRC Press, New York

[102] Zhou M, Centeno V, Thorp J (2006) An alternative for including phasor measurements in state estimators. IEEE Trans Power Syst 21(4):1930-1937

[103] Meliopoulos A, Zhang F (1996) Multiphase power flow and state estimation for power distribution systems. IEEE Trans Power Syst 11(2):939-946
[104] Heydt G (2010) The next generation of power distribution systems. IEEE Trans Smart Grid 1(3):225-235

[105] Liu J, Tang J, Ponci F et al (2012) Trade-offs in PMU deployment for state estimation in active distribution grids. IEEE Trans Smart Grid 3(2):915-924

[106] Hu Y, Kuh A, Kavcic A et al (2011) Real-time state estimation on micro grids. In: Proceedings of international joint conference on neural networks, San Jose, USA, 31 July-5 August 2011, 8 pp

[107] Albu M, Kyriakides E, Dumitrescu A et al (2011) Analysis of distribution grids: state estimation using model uncertainties. In: Proceedings of IEEE international workshop on applied measurements for power systems, Aachen, Germany, 28-30 September 2011, 6 pp

[108] Florea I, Naumof M, Dumitrescu A (2011) Uncertainty budget for distribution grids analysis: a study case on model parameters. In: Proceedings of 7th international symposium on advanced topics in electrical engineering, Bucharest, Romania, 12-14 May 2011, 6 pp

[109] Chai WK, Wang N, Katsaros KV (2015) An information-centric communication infrastructure for real-time state estimation of active distribution networks. IEEE Trans Smart Grid 6(4):2134-2146

[110] Enslin J, Heskes P (2004) Harmonic interaction between a large number of distributed power inverters and the distribution network. IEEE Trans Power Electron 19(6):1586-1593

[111] Carta A, Locci N, Muscas C (2009) A PMU for the measurement of synchronized harmonic phasors in three-phase distribution networks. IEEE Trans Instrum Meas 58(10):3723-3730

[112] Ayala G, Aguerc J, Elizondo D et al (2013) Current trends on applications of PMUs in distribution systems. In: Proceedings of IEEE PES innovative smart grid technologies conference, Washington, USA, 24-27 February 2013, 6 pp

[113] Alinejad B, Akbari M, Kazemi H (2012) PMU-based distribution network load modeling using harmony search algorithm. In: Proceedings of 17th conference on electrical power distribution, Tehran, Iran, 2-3 May 2012, 6 pp

[114] Xu Y, Si D, Qian Y (2011) Research on feasibility of composite load modeling based on WAMS. In: Proceedings of AsiaPacific power and energy engineering conference, Wuhan, China, 25-28 March 2011, 4 pp

[115] Ledwich G, Moyano C (2011) Synchrophasors for load modelling in Australia. In: Proceedings of IEEE PES general meeting, Detroit, USA, 24-29 July 2011, 6 pp

[116] Meier A, Culler D, McEachern A (2014) Micro-synchrophasors for distribution systems. In: Proceedings of ISGT, Washington, USA, 19-22 February 2014, 5 pp

[117] Meier A, Stewart E, McEachern et al (2017) Precision microsynchrophasors for distribution systems: a summary of applications. IEEE Trans Smart Grid 8(6):2926-2936

[118] Pinte B, Quinlan M, Reinhard K (2015) Low voltage microphasor measurement unit (Micro-PMU). In: Proceedings of IEEE power and energy conference at Illinois, Champaign, USA, 20-21 February 2015, 4 pp

[119] Peterson S, Whitacre J, Apt J (2010) The economics of using plugin hybrid electric vehicle battery packs for grid storage. J Power Sources 195:2377-2384

[120] Usman MU, Kelle D, Baldwin T (2014) Utilizing spinning reserves as energy storage for renewable energy integration. In: Proceedings of Clemson University power systems conference, Clemson, USA, 11-14 March 2014, 5 pp 
Muhammad Usama USMAN is a Ph.D. candidate in Electrical Engineering at Florida State University, USA. He received the M.Sc. degree in Electrical Engineering from Florida State University, USA in 2014. He served as a Lecturer with the Department of Electrical Engineering at UAJK, Pakistan from 2014 to 2015 . He is presently working as a Research Assistant with Center for Advanced Power Systems, Florida State University. His current research interests include synchrophasor technologies and data analytics in power systems, outage management system and development of advanced metering infrastructure for smart grids.
M. Omar FARUQUE received the Ph.D. degree from the University of Alberta, Edmonton, AB, Canada, in 2008, and since then he has been working with the Department of Electrical and Computer Engineering at FAMU-FSU College of Engineering, Florida State University, Tallahassee, USA, and the Center for Advanced Power Systems. His research areas include modeling and simulation (offline and real-time), smart grid and renewable energy integration, allelectric-ship power system, and hardware-in-the-loop-based experiments. 